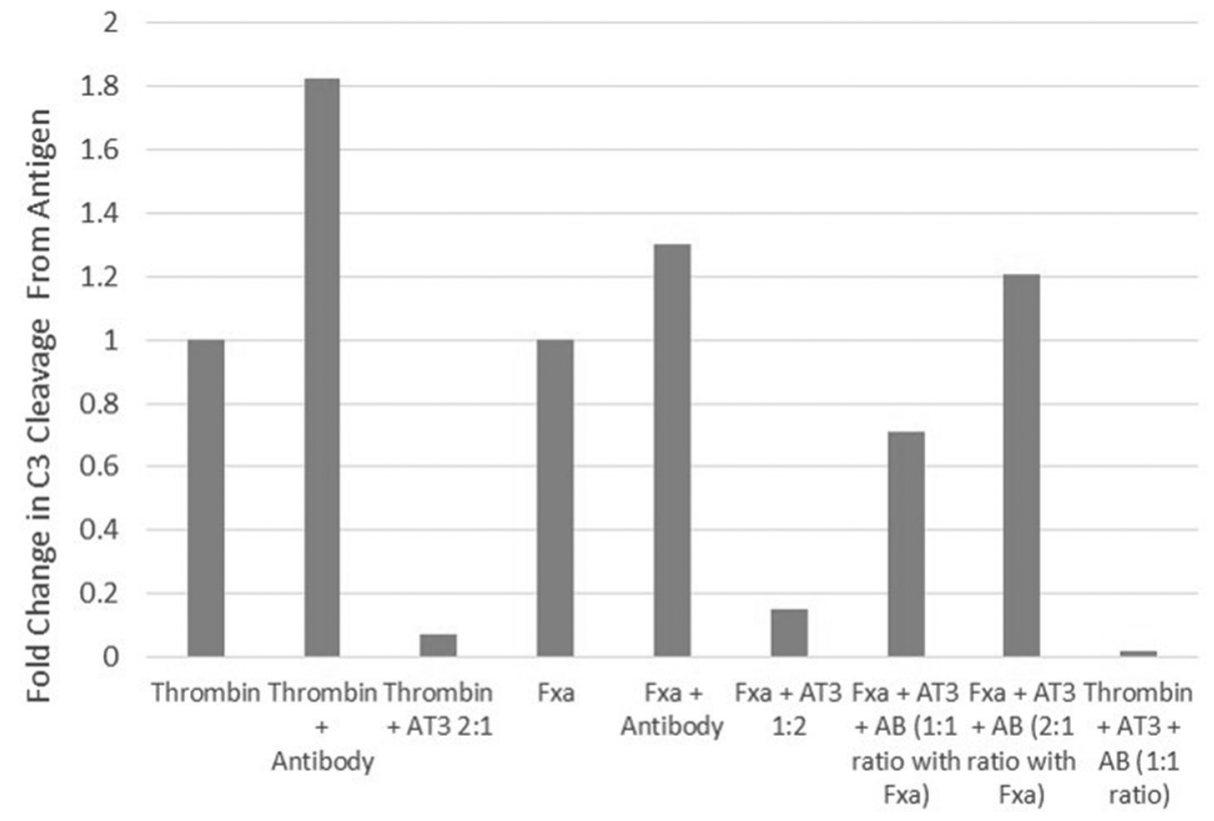

Abstract PS5:99 Figure 1 Data showing relative C3 cleavage of Thr and FXa in the presence and absence of antibodies and inhibitors

$\operatorname{IgG}(n=2)$ but not anti-Thr IgG. Analysis of clinical serology records for the last 10 consecutive clinic visits of 28 anti-SPpositive patients showed a lower level of C3 $(0.92 \mathrm{~g} / \mathrm{L})$ in the patients double positive for anti-FXa and anti-Thr than for anti-Thr alone $(1.12 \mathrm{~g} / \mathrm{L})$ or anti-FXa alone $(1.16 \mathrm{~g} / \mathrm{L})$.

Conclusions Anti-FXa and anti-Thr enhance cleavage of C3 by FXa and Thr respectively. Presence of these antibodies in-vivo in patients with SLE and/or APS may promote increased complement activation and disease activity. This finding may have potential translational implications for future treatment of these diseases.

\section{PS5:100 PATOPHYSIOLOGICAL ROLE OF TYPE I AND III INTERFERONS IN SYSTEMIC LUPUS ERYTHEMATOSUS} (SLE)

${ }^{1} \mathrm{M}$ Meineck, ${ }^{1} \mathrm{~A}$ Kommer, ${ }^{2} \mathrm{~L}$ Schmidtke, ${ }^{1} \mathrm{~J}$ Lutz, ${ }^{1} \mathrm{~A}$ Schwarting, ${ }^{2} \mathrm{~A}$ Pautz, ${ }^{1} \mathrm{~J}$ WeinmannMenke. ${ }^{1}$ Department of medicine, University medical centre of the Johannes Gutenberg University, Mainz, Germany; ${ }^{2}$ Department of Pharmacology, University medical centre of the Johannes Gutenberg University, Mainz, Germany

\subsection{6/lupus-2018-abstract. 145}

Systemic Lupus erythematosus (SLE) is an autoimmune disease characterised by activated autoreactive lymphocytes and autoantibodies, resulting in tissue damage in multiple organs. An important factor for the disease's mortality is the development of Lupus nephritis (LN). Type I and III interferons, which are both part of the antiviral defense, have both been associated with the disease's activity. In sera and urine of SLE patients an enhanced level of IL28/29 was described, but their distinct functional role in the course of disease need to be further investigated.

To determine the role of type I and III interferons during onset and progression of autoimmunity - with focus on the development of $\mathrm{LN}$ - the expression of the IFNs and their specific receptors was observed in lupus prone MRL Faslpr mice. These mice develop SLE like symptoms and immunocomplex glomerulonephritis. So far we could confirm the expression of IL28 and it's receptor by tubular epithelial cells
(TEC) in the kidney of MRL Faslpr mice. The overall IL28 mRNA expression increased with disease activity in renal tissue, and a positive correlation to the IFN $\alpha$ and IFN $\beta$ expression could be observed. Further the mRNA expression of the IFN receptor mRNA in the spleen accelerated with increasing disease activity.

Furthermore MRL Faslpr mice deficient of the IL28R and/or IFN $\alpha$ R were generated and the progression of autoimmunity and LN was monitored. In preliminary studies with MRL Faslpr IL28R -/- mice, a less extenuated lymphadenopathy and less severe $\mathrm{LN}$ at the age of 3 month was observed, compared to their wildtype littermates. Similar observations according the Lymphadenopathy were made in MRL Faslpr IFN $\alpha$ R -/- mice.

Our results suggest a participation of type III IFNs in the development of Lupus nephritis in MRL Faslpr mice. In upcoming experiments the effect of the IL28R knockout will be compared to the effect of the IFN $\alpha \mathrm{R}$ knockout and the combined IL28R-IFN $\alpha$ R knockout. The subsequent aim is to transfer the results obtained in the murine model to human SLE and to evaluate IL28 as disease activity marker.

\section{PS5:101 NUCLEAR ANTIGEN-REACTIVE CD4+ T CELLS ARE EXPANDED IN ACTIVE SLE, CORRELATE WITH DISEASE ACTIVITY, INVADE TARGET ORGANS SUCH AS THE KIDNEYS, AND OUTNUMBER THEIR REGULATORY COUNTERPARTS}

\footnotetext{
1,2D Abdirama, 1,2S Tesch, 1,2A-S Griessbach, ${ }^{1,2} \mathrm{~A}$ Scheffold, ${ }^{2} \mathrm{~A}$ Radbruch, ${ }^{3,4} \mathrm{U}$ Stervbo, ${ }^{3,4} \mathrm{~N}$ Babel, ${ }^{1} \mathrm{G}-\mathrm{R}$ Burmester, ${ }^{5} \mathrm{JY}$ Humrich, ${ }^{5} \mathrm{G}$ Riemekasten, ${ }^{6} \mathrm{P}$ Enghard. ${ }^{1}$ Charité Universitätsmedizin Berlin Department of Rheumatology and Clinical Immunology, Berlin, Germany; ${ }^{2}$ Deutsches Rheuma-Forschungszentrum a Leibniz Institute, Berlin, Germany; ${ }^{3}$ Berlin-Brandenburg Centre for Regenerative Therapy, Berlin, Germany; ${ }^{4}$ Universitätsklinikum der Ruhr-Universität Bochum Centre for Translational Medicine, Bochum, Germany; ${ }^{5}$ Universitätsklinikum Schleswig-Holstein Department of Rheumatology and Clinical Immunology, Lübeck, Germany; ${ }^{6}$ Charité Universitätsmedizin Berlin Department of Nephrology and Intensive Care Medicine, Berlin, Germany
}

10.1136/lupus-2018-abstract.146 
Background and objectives Loss of tolerance towards nuclear antigens such as SmD1, RNP70, Histone, Ro, and La due to defective defective disposal of biological waste is characteristic of SLE. The question whether CD4 $+\mathrm{T}$ cells reactive to the aforementioned autoantigens are also implicated in SLE remains unclear. To study the role of autoreactive CD4 +T cells in SLE, we determined their frequency in healthy and diseased state, investigated their antigen specificity, characterised their cytokine production, interrogated their localization in the tissue, and analysed their homeostatic balance with autoreactive regulatory $\mathrm{T}$ cells (Treg).

Materials and methods We employed: $\mathrm{T}$ cell library to detect peripheral nuclear autoantigen-reactive CD4 +T cells, antigenreactive $\mathrm{T}$ cell-enrichment to interrogate their cytokine production, generation of $\mathrm{T}$ cell clones and $\mathrm{T}$ cell lines to validate their specificity, TCR-beta analysis to predict their clonal occurence in blood and urine, urinary $\mathrm{T}$ cell library to detect and enumerate their frequencies in urine, CFSE-labelled urinary cells to detect and enumerate their frequencies in urine, and enrichment of CD137 +FoxP3 + cells to investigate nuclear antigen-reactive Treg.

Results The frequency of autoreactive CD4 $+\mathrm{T}$ cells is greater in active SLE patients when compared with inactive SLE patients and healthy individuals, and it correlates with disease activity. Single-cell clones of autoreactive CD4 +T cells confirmed their antigen specificity. Autoreactive CD4 +T cells produced pro-inflammatory cytokines such as IFN-gamma, IL17, and IL-10, where the production of IFN-gamma correlates with disease activity. The accumulation of these cells was detected in urine of active SLE patients with lupus nephritis when compared with their number in the periphery. The ratio of autoreactive effector $\mathrm{T}$ cells against autoreactive Treg is higher in active SLE patients when compared with inactive SLE patients and healthy individuals, and it correlates with disease activity.

Conclusions Our data demonstrate that higher frequency of autoreactive CD4 $+\mathrm{T}$ cells in the periphery could contribute to disease progression of SLE. Accumulation of these cells in urine suggested their possible role in mediating local tissue inflammation. Homeostatic imbalance between autoreactive effector $\mathrm{T}$ cells and Treg indicated an insufficient regulatory mechanism to control autoimmunity at antigen-specific level.

\section{PS5:102 THE EXPRESSION OF BTLA ON CMV-SPECIFIC T-CELLS IS DECREASED IN CMV IGG+ PATIENTS WITH SYSTEMIC LUPUS ERYTHEMATOSUS}

${ }^{1} \mathrm{~A}$ Savidis, ${ }^{1} \mathrm{~B}$ Wilde, ${ }^{1} \mathrm{~A}$ Kribben, ${ }^{2} \mathrm{O}$ Witzke, ${ }^{2} \mathrm{~S}$ Dolff. ${ }^{1}$ Department of Nephrology, University Hospital Essen, Germany; ${ }^{2}$ Department of Infectious Diseases, University Hospital Essen, Germany

\subsection{6/lupus-2018-abstract. 147}

Introduction Cytomegalovirus (CMV) infection is an uncommon but severe infection in patients with systemic lupus erythematosus (SLE) due to immunosuppressive therapy. Standard prophylaxis with antiviral agents or pre-emptive strategies to monitor viral load are not standard of care. The aim of the present study was to investigate the expression of coinhibitory molecules PD-1 and BTLA-4 on CMV specific T-cells in SLEpatients.
Methods Sixteen SLE-patients and eight healthy controls were enrolled. Tweleve SLE patients were CMV IgG+, four were CMV IgG-. Peripheral blood was sampled and stimulated with CMV lysate, SEB or control serum in presence of anti-CD28/ CD49d. After six hours of stimulation, CD154 expression was determined by flow cytometry on CD3 + T cells. The coinhibitory molecules PD-1 and BTLA were determined on activated CD154+CD3+T cells. Symptomatic CMV infection was defined as CMV syndrome or tissue invasive disease. Asymptomatic CMV infection was defined as detectable CMV replication in peripheral blood and absence of signs indicating CMV syndrome/tissue invasive disease.

Results PD-1 and BTLA-4 expression was not significantly different on CMV-specific CD154 +CD3+T cells in SLE-patients as compared to healthy controls. An analysis according to the CMV serostatus revealed a tendency to a decreased proportion of PD-1 +CD154+CD3+T cells in CMV IgG negative patients as compared to CMV IgG positive $(24.9 \% \pm 30.0 \%$ vs $35.5 \pm 11.5 \%, \mathrm{p}=0.3)$. The BTLA-4 expression was significantly decreased on CD154 +CD3+T cells in CMV IgG negative patients as compared to $\mathrm{CMV}$ IgG positive $(70.3 \%$ $\pm 31.7 \%$ vs $95.0 \pm 4.3 \%$, $\mathrm{p}=0.01$ ).

Conclusion SLE-patients show a significant decreased expression of BTLA on CMV-specific T-cells. The coinhibitors PD-1 and BTLA usually promote T-cell suppression. Thus a decrease may prone to severe symptomatic infections.

\section{PS5:103 COSTIMULATORY MAKER ON CYTOTOXIC CD8+ T- CELLS IN PATIENTS WITH SLE}

${ }^{1} \mathrm{C}$ Oster, ${ }^{1} \mathrm{~B}$ Wilde, ${ }^{1} \mathrm{~K}$ Hübbers, ${ }^{1} \mathrm{~A}$ Kribben, ${ }^{2} \mathrm{O}$ Witzke, ${ }^{2} \mathrm{~S}$ Dolff. ${ }^{1}$ Department of Nephrology, University Hospital Essen, Germany; ${ }^{2}$ Department of Infectious Diseases, University Hospital Essen, Germany

\subsection{6/lupus-2018-abstract.148}

Background Systemic lupus erythematodes (SLE) is an autoimmune disease where T-cell dependent B-cell activation plays a crucial role. Costimulatory marker promote and inhibit immune responses after ligation. Here we analysed the marker BTLA (CD272), PD-1 (CD279), HLA-DR and HVEM on peripheral cyctotoxic T-cells.

Patients and methods 30 SLE-patients fulfilling the American College of Rheumatology (ACR) criteria für SLE and 14 healthy controls (HC) were enrolled. Whole blood staining was performed and samples were analysed by multi-colour flow cytometry. Disease activity was assessed by SLEDAI. Renal involvement was defined as biopsy proven lupus nephritis.

Results The expression of the costimulatory marker PD-1 is signifcantly increased on CD8 + Tcells in SLE patients as compared to with healthy controls $(31.3 \% \pm 26.7 \%$ vs $15.63 \%$ $\pm 12.22 \%$ ). We could not found a significant difference in the expression levels of the BTLA receptor on CD8 +T cells $(72,24 \% \pm 15.33$ vs $72.66 \% \pm 9,4 \%)$ in SLE patients as compared to with healthy controls. Also HLA-DR and HVEM was not significantly different expressed on CD8 +T cells in SLE.

Conclusions The costimulytory maker PD-1 is upregualted on cytotoxic T-cells in SLE. Further investigations are needed to elucidate the functional role of this pattern. 